\section{Revue d'ethnoécologie}

$20 \mid 2021$

Un siècle d'ethno-machins : le centenaire de la Revue de Botanique Appliquée

\title{
Essai cartographique de la production bibliographique en ethnobotanique au Brésil
}

Mapping the ethnobotanic bibliographic production in Brazil

\section{Laure Emperaire}

\section{OpenEdition}

Journals

Édition électronique

URL : https://journals.openedition.org/ethnoecologie/8102

DOI : 10.4000/ethnoecologie.8102

ISSN : 2267-2419

Éditeur

Laboratoire Éco-anthropologie

Référence électronique

Laure Emperaire, «Essai cartographique de la production bibliographique en ethnobotanique au Brésil », Revue d'ethnoécologie [En ligne], 20 | 2021, mis en ligne le 31 décembre 2021, consulté le 06 mars 2022. URL : http://journals.openedition.org/ethnoecologie/8102 ; DOI : https://doi.org/10.4000/ ethnoecologie.8102

Ce document a été généré automatiquement le 6 mars 2022.

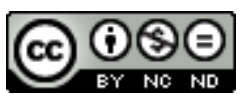

Revue d'ethnoécologie est mis à disposition selon les termes de la licence Creative Commons Attribution - Pas d'Utilisation Commerciale - Pas de Modification 4.0 International. 


\title{
Essai cartographique de la production bibliographique en ethnobotanique au Brésil
}

Mapping the ethnobotanic bibliographic production in Brazil

\author{
Laure Emperaire
}

\section{Introduction}

1 Si, comme le disait A.-G. Haudricourt, cité par J. Barrau dans ses cours, «l'ethnobotanique est la botanique des autres» phrase restée célèbre, l'ethnobotanique a une trajectoire qui se perd dans le regard que chaque peuple est susceptible de porter sur les connaissances et les pratiques du végétal des autres. Aujourd'hui, ce que l'on sait des plantes ou de l'univers végétal des autres traverse une vaste panoplie de champs disciplinaires, d'objectifs et d'enjeux. À l'échelle mondiale, la matérialité végétale des relations avec les sociétés humaines, un support évident de l'ethnobotanique jusqu'à il y a peu, devient évanescent. Ainsi, une ethnographie centrée sur les DSI (Digital sequence information), ou, en français les «données numériques de séquences de ressources génétiques » (FRB 2019), un enjeu fort des négociations de la COP 15 de 2022, pourrait relever d'une ethnobotanique imprégnée du droit et de bio-informatique en contre-point de celle porteuse de visions chamaniques et de relations apaisées avec l'environnement. Les deux nous projettent des mondes à venir.

2 Le thème en filigrane de cet article est celui de la trajectoire de l'ethnobotanique dans le contexte du Brésil, pays central dans ces débats de par sa diversité biologique et culturelle. L'article est constitué de trois parties. La première porte sur le processus historique de construction de ce qui deviendra l'ethnobotanique à partir de publications qui s'étendent de la période coloniale à celle de la formation de l'espace scientifique brésilien. La seconde va de la fin du dix-neuvième siècle à aujourd'hui et prend en compte deux forces majeures, la consolidation des institutions scientifiques et 
la diffusion des connaissances produites et leur intégration dans des disciplines scientifiques. Ces deux premières parties reposent sur une analyse, non exhaustive, de la bibliographie. La troisième partie traite des configurations actuelles de l'ethnobotanique. Elle résulte de la consultation de bases de données brésiliennes et présente un panorama général de la production scientifique et des profils scientifiques des chercheurs brésiliens investis dans l'étude des relations plantes-sociétés. Il est important de préciser que l'angle d'analyse n'est pas celui d'une histoire des sciences mais celui d'un regard d'ethnobotaniste qui participe à la marge de cette trajectoire.

\section{Entre raison d'État et exotisme}

Difficile de démêler dans la trajectoire de l'ethnobotanique au Brésil ce qui relève des prémices de la connaissance scientifique des végétaux, de l'émergence d'une histoire naturelle, de l'apprentissage d'un nouvel espace de vie, d'une curiosité pour un exotisme et d'une convoitise évangélisatrice et économique. Le contexte est celui de l'invasion de nouvelles terres et la raison d'État n'est jamais loin, qu'elle soit ibérique ou hollandaise (ou encore française avec les écrits de Thevet, de Léry, d'Abbeville ou d'Évreux). En 1557, Hans Staden livre une première description de manioc, de ses dérivés et de quelques autres plantes chez les Tupinamba (Staden 2010 [1557]). L'História dos animais e árvores do Maranhão de Frei Cristovão Lisboa (Lisboa 1967 [1625]) rédigée et illustrée entre 1625 et 1631, mais qui ne sera publié qu'en 1967 au Portugal, précède de peu l'Historia Naturalis Brasiliae de Marcgrave et Pison publiée en 1648 par Elsevier (Marcgrave \& Pison 1648). Une riche iconographie accompagne ces ouvrages. Les données sont issues d'observations in situ comme le souligne Malheiros (2013) et apportent un éclairage global sur la phytonymie, les usages - en particulier médicinaux -, et l'écologie des plantes des régions nord et nordeste du Brésil. Une ethnobotanique descriptive, pratique et économique se dessine. Avec Frutas do Brasil numa nova, $e$ ascetica Monarchia, consagrada à Santissima Senhora do Rosário du Frère Antônio do Rosário en 1702, l'inventaire des fruits, locaux ou introduits, se mue en un instrument politique pour dénoncer la structure de la société et l'abandon dans lequel la couronne portugaise laisse le pays (Almeida 2002). Aujourd'hui, l'ouvrage de Marcgrave et Pison est revendiqué comme étant au fondement de la botanique brésilienne. Celui de Gabriel Soares de Sousa Traité descriptif du Brésil de 1587 pourrait l'être quant à l'agronomie brésilienne : diversité variétale des plantes cultivées majeures et modes d'usage y sont décrits avec une précision qu'il sera difficile de retrouver plus tardivement (Soares de Sousa 1851 [1587]).

4 Le Jésuite João Daniel, d'origine portugaise, apporte l'une des plus importantes contributions du dix-huitième siècle à la connaissance de l'Amazonie où il séjournera quinze ans. Expulsé du pays en 1758 comme tous les Jésuites, il est incarcéré près de vingt ans à son retour au Portugal. Il écrira de sa prison le Tesouro descoberto no Rio Máximo Amazonas, 800 pages de descriptions détaillées sur tous les aspects de la vie et des environnements amazoniens, des aspects triviaux de la vie en Amazonie avec ses acariens qui vous démangent à ses paysages et richesses (Daniel 2004 [1757-1776]). Les plantes, cultivées ou forestières sont énumérées et commentées en adoptant la perspective du pouvoir politique en place. Ainsi, la culture du manioc est critiquée par rapport à celle des céréales qui auraient l'intérêt de fixer durablement l'homme à la terre, objectif du pouvoir en place (Carneiro da Cunha 2019) - ce qui répond encore 
aujourd'hui à une conception de l'agriculture fréquemment portée par les politiques publiques. Dans la seconde moitié du dix-huitième siècle, l'enjeu est d'approfondir les connaissances sur les ressources végétales locales afin de promouvoir leur mise en culture. Des jardins botaniques sont créés à Rio de Janeiro, São Paulo, Belém, Ouro Preto et Salvador pour accompagner ces expériences et la mise en plantation d'espèces introduites (Dean 1991).

5 Au dix-neuvième siècle, la perspective des voyageurs naturalistes mandatés par leurs pays domine. Il s'agit de collecter et de décrire au plus près afin de rendre compte d'une réalité qui imprègne les sens et à laquelle se mêle une emphase romantique mais avec un souci de fidélité toujours présent (Kury 2001). Nombre de descriptions portent sur les usages des plantes et les techniques utilisées. Mais l'importance donnée aux compte-rendu de ces voyageurs (Martius, Saint-Hilaire et autres) éclipse en partie la production scientifique brésilienne, principalement botanique, du dix-neuvième siècle. Ainsi, les 65 collaborateurs de la Flora brasiliensis de Martius, originaires de neuf pays européens, ne laissent pas de place aux collaborations brésiliennes. Dans son étude historique sur la zoologie et la botanique au Brésil, Neiva (1929) souligne combien João Barbosa Rodrigues (1842-1909), spécialiste des palmiers et des orchidées et premier directeur du Jardin Botanique de Rio de Janeiro aurait pu substituer Oscar Drude, botaniste allemand, pour les premiers ou Célestin A. Cogniaux, botaniste belge, pour les secondes. La réquisition des 1134 planches d'herbier du voyageur naturaliste portugais, Alexandre Ferreira Rodrigues par le général Junot en 1808 à la demande de SaintHilaire est un épisode connu (Telles Antunes 2011). Ces herbiers seront néanmoins restitués en 1815. La trajectoire de ce que deviendra l'ethnobotanique au Brésil est une suite d'ajustements, un équilibre instable entre intérêt pour d'autres savoirs, enjeux géostratégiques, enjeux économiques et savoirs et prestiges scientifiques.

\section{La constitution de corpus de données ethnobotaniques}

$6 \quad$ Les deux chapitres du dernier volume du Handbook of South American Indians édité par Julian Steward de 1946 à 1950 constituent les premières synthèses les rapports plantessociétés en Amérique du Sud. Lévi-Strauss (1950) met l'accent sur le complexe formé par la plante sauvage, ses techniques d'exploitation et la culture matérielle associée et insiste sur les complémentarités entre les sphères du spontané et du cultivé. Sauer (1950) y interroge la distribution des plantes cultivées, espèces ou variétés pour certaines, leurs voies de diffusion et leurs usages. Ces contributions, traduites en portugais, constitueront le volet ethnobotanique du premier volume de la Suma Etnológica Brasileira coordonné par Berta Ribeiro (1986), anthropologue, spécialiste de la culture matérielle et professeure au Département d'Anthropologie du Museu Nacional à Rio de Janeiro. Ce volume comprendra aussi des articles issus du volume 5 The Comparative Ethnology of South American Indians du Handbook ainsi que des contributions plus récentes d'autres auteurs fondateurs de l'ethnobotanique brésilienne. Les thématiques développées par des chercheurs de diverses disciplines (pharmacologie, anthropologie, agriculture, génétique) sont pionnières, et toujours d'actualité (domestication et sélection des plantes cultivées, gestion des unités de paysage, réseaux de circulation des plantes, plantes médicinales). C'est aussi la période du lancement de la série des Advances in Economic Botany édité par le New York Botanical Garden et, un 
peu plus tard, celledu développement des approches quantitatives développées par Prance, Balée et Carneiro (1987), reformalisées par la suite par Phillips et al. (1994). Ces publications s'inscrivent dans les débats sur la conservation de la biodiversité telle qu'elle est portée depuis 1992 par la Convention sur la Diversité Biologique.

Une autre approche est développée par Victor M. Patiño, agronome et botaniste colombien. Celui-ci établit un recensement des citations des plantes cultivées dans les sources historiques et offre par là des pistes de recherche sur la diffusion des plantes cultivées en Amérique équinoxiale (Patiño 1963-1974). Sa principale œuvre est composée de cinq volumes, publiés entre 1963 et 1974, qui portent sur les fruitiers, les plantes alimentaires, celles à fibres, celles médicinales et à usages divers, celles introduites, les animaux domestiques et enfin les plantes ornementales. Une quinzaine d'années plus tard, V. M. Patiño (1989) publiera une Bibliografia etnobotanica parcial comentada de Colombia y paises vecinos qui comporte 702 références sur la région équatoriale dont 40 sur le Brésil et 59 sur l'Amazonie en général. Chaque source est brièvement commentée en identifiant la société concernée, les plantes, ou la culture matérielle associée.

Où placer une autre œuvre monumentale, celle de Pio Corrêa, botaniste portugais qui a publié entre 1926 et 1952 un Dictionnaire des plantes utiles et des exotiques cultivées du Brésil en six volumes de chacun plus de 500 pages (Corrêa 1984 [1926-1952] ? Ses notes explicatives indiquent que l'ouvrage a été rédigé sur la base d'observations de terrain recueillies au Brésil et dans de nombreux autres pays, d'échanges au sein des réseaux scientifiques et d'une compilation de la bibliographie. Les entrées par nom vernaculaire sont suivies du ou des noms scientifiques, d'une description de la plante, de ses usages et de sa répartition. Un index des noms scientifiques clôt chaque volume. L'absence de bibliographie, dont la publication aurait été selon l'auteur «fastidieuse » tout comme celle d'un index commun aux six volumes en font, malgré la somme d'informations exposées, un ouvrage de consultation parfois malaisée.

Le Museu Paulista à São Paulo constitue le point d'ancrage du rapprochement entre une ethnobotanique en construction et les sciences humaines et sociales. Ce musée, fondé en 1895, a d'abord été un musée d'histoire naturelle au caractère encyclopédique marqué (Françozo 2005) avant de mettre l'accent, en 1922, sur l'histoire du Brésil et de l'État de São Paulo. Sa section de botanique est transférée dès 1927 dans une autre institution et la rédaction du premier recensement bibliographique sur l'ethnobotanique au Brésil est confiée à Beulah Coe-Teixeira, botaniste spécialiste des Lauracées attaché à l'Institut de Botanique du Secrétariat de l'Agriculture de São Paulo. La Bibliografia Comentada de Etnobotânica est publiée en 1976 dans la revue du Musée de São Paulo ou Museu Paulista (Coe-Teixeira 1976). Son objectif est d'appuyer le développement de la "recherche en ethnobotanique dans le Secteur d'Ethnologie du Museu Paulista de l'Université de São Paulo » (ibid. p.45). L'auteur reconnaît que, avec une soixantaine de références citées, la portée de son recensement de travaux est limitée, mais sa préoccupation est de recenser au plus tôt des savoirs et une flore. Les premières lignes de son introduction en témoignent de manière tout à fait actuelle (ibid. p. 43) :

«Our flora is being destroyed so fast that we have no way of knowing what existed before. The only hope lies in the possibility of convincing the men who have the power of decision to help before it is too late ».

Ses notes et commentaires sur l'usage des plantes sont en partie issus de l'ouvrage de Herbert Baldus, ethnologue rattaché au Museu Paulista qui a publié en deux volumes 
une Bibliografia crítica da etnologia brasileira $(1954,1968)$. Ils seront complétés en 1984 par un troisième volume rédigé par Tekla Hartmann, autre ethnologue du Museu Paulista (Hartmann 1984). La Bibliografia crítica da saúde indígena no Brasil (1844-2006) établie par D. Buchillet (2007), anthropologue de la santé qui a intensément défendu une approche multiculturelle des soins, participe aussi de cette mise en ordre des connaissances sur le végétal : plus de 3000 références commentées y sont compilées dont 380 concernent des plantes en rapport avec l'alimentation ou les soins.

11 L'ethnobotanique ne se fond pas pour autant dans le développement de l'anthropologie au Brésil et demeure un champ à part. La remarque introductive de B. Ribeiro dans la Suma Etnológica Brasileira (Ribeiro 1986: 12) va dans ce sens : " De fait, l'ethnobiologie est une science nouvelle au Brésil cultivée, malheureusement, par peu d'anthropologues » mais elle pourrait être aussi lue en en inversant les termes, bref deux courants se dégagent avec cependant des points de jonction dans les domaines de la conservation de la biodiversité et la reconnaissance des droits territoriaux et intellectuels des populations traditionnelles.

\section{Ethnobotanique et populations traditionnelles}

Le contexte politique et écologique des années 1980-1990 imprime une nouvelle dimension à ces recherches. La Nouvelle Constitution est promulguée en octobre 1988. Son article 231 stipule que «Sont reconnus aux Indiens leurs organisation sociale, coutumes, langues, croyances et traditions, et les droits originaires sur les terres qu'ils occupent traditionnellement, étant de la compétence de l'Union de les démarquer, protéger et faire respecter tous leurs biens " [notre traduction, Brasil 1988]. La même année, un collectif de chercheurs et de représentants des populations traditionnelles rédige la déclaration de Belém de $1988^{1}$, pierre fondamentale de la reconnaissance de l'importance des savoirs autochtones sur la biodiversité et du nécessaire dédommagement pour leur utilisation, deux éléments clé de la Convention sur la Diversité Biologique et de la Déclaration de Rio de Janeiro de 1992. C'est la période de nouvelles convergences entre chercheurs engagés dans la conservation des territoires traditionnels et d'alliances entre peuples indigènes et communautés traditionnelles (d'où l'Alliance des Peuples de la Forêt). En même temps, se dessinent de nouvelles visées marchandes, autour de l'exploitation des ressources forestières et des savoirs associés. C'est un "or vert » qui est attendu des substances naturelles, mais l'expectative ne se concrétisera pas, même après trente ans (Aubertin 2021). Cependant de nouvelles configurations territoriales qui renouvellent les conceptions de la conservation de la biodiversité résulteront de ces articulations chercheurs, politiques et populations locales. Le décret sur les Réserves Extractivistes est promulgué en 1990 et, en 2000, les Réserves de Développement Durable sont instituées (Brasil 1990 ; Brasil 2000).

13 Le processus de création de la Réserve Extractiviste du Haut Juruá (REAJ) suscite de nouvelles articulations, ici avec les seringueiros ${ }^{2}$, en matière de recherche et de publications. Une recherche pilote sur les locaux sur la forêt est lancée en 1993 par M. Almeida, M. Carneiro da Cunha, K. Brown et l'Association locale des Seringueiros et Agriculteurs de la REAJ. Chercheurs anthropologues, biologistes, géologues, étudiants, seringueiros et Amérindiens Kaxinawá, Katukina et Ashaninka entreprennent de rendre compte de l'étendue des savoirs sur la forêt du Haut Juruá, dans l'état d'Acre. Les recherches seront publiées dans l'Encyclopédie de la Forêt en 2002, un ouvrage de 735 
pages qui a compté sur plus d'une centaine de collaborations et qui rend compte à la fois de la connaissance encyclopédique locale du milieu et de l'expérience de coproduction de savoirs (Carneiro da Cunha \& Almeida 2002). Vingt ans plus tard, c'est une autre publication de synthèse qui est mise en ligne Contributions des Peuples amérindiens, des Quilombolas et des Communautés traditionnelles à la biodiversité, politiques et menaces (Carneiro da Cunha, Barbosa Magalhães et Adams 2021). En 17 volumes, elle s'affranchit de la seule perspective de la recherche scientifique, fait dialoguer chercheurs non académiques et académiques, et trois volumes (Neves 2021, Emperaire 2021, Morim de Lima, Cabral de Oliveira et Shiratori 2021) traitent directement des relations à l'environnement.

14 En parallèle, les publications des détenteurs des connaissances sur la biodiversité s'étoffent. Elles dessinent un nouveau courant élargi de la production de savoirs sur les relations société-environnement. Difficile encore de les recenser car elles sont dans de multiples circuits de production, associations amérindiennes, ONG, ou publications d'initiative individuelle. Cette dynamique est aussi sous-tendue par la réalisation de travaux universitaires de jeunes chercheurs amérindiens, quilombolas ${ }^{3}$ ou de communautés traditionnelles (Silva 2013, Barretto 2021 entre autres).

\section{L'ethnobotanique au prisme des bases de données académiques et scientifiques}

15 Sur le plan de l'enseignement, l'ethnobotanique, considérée en tant que discipline, n'apparaît pas dans les formations en sciences sociales et humaines. Elle est rattachée selon les universités aux sciences biologiques et écologiques, pharmaceutiques, agraires, forestières ou encore en développement durable avec des charges horaires allant de 30 à 60 heures en équivalent licence et master et de 45 à 120 heures en doctorat (Fonseca-Kruel et al. 2005). Treize universités proposent un enseignement spécifique dans ce domaine. Les priorités générales de ces enseignements, identifiées à partir d'un questionnaire, sont la standardisation des méthodes, la mise en œuvre d'approches quantitatives et l'adaptation de méthodes d'échantillonnage. Elles visent à renforcer la rigueur quantitative des analyses sur la base d'hypothèses formalisées. En marge de ces objectifs de connaissance, de nombreux travaux d'ethnobotanique ou de botanique économique sont réalisés dans une perspective de mise en valeur de ressources biologiques.

16 Les syllabus des cours d'anthropologie sociale et culturelle montrent une approche différente des rapports à l'environnement (par exemple la Construction des concepts de nature et de société en anthropologie à l'Université de São Paulo où les relations humains non humains, les réseaux de savoirs tiennent une place importante, ou encore Anthropologie de l'Environnement, cultures végétales en Amazonie au Museu Nacional de l'Université Fédérale de Rio de Janeiro. Le département d'Anthropologie de l'Université Fédérale d'Amazonas (UFAM) propose le thème Domestications Amazoniennes: approches anthropologiques, archéologiques et d'écologie historique. Le thème dialogue avec ceux de l'Institut National de Recherches en Amazonie (INPA ${ }^{4}$ ) qui propose dans les cursus d'écologie et de botanique des enseignements en ethnobiologie, en botanique économique et aborde les rapports environnement - société à travers les domestications. Les cadres d'une interdisciplinarité élargie s'ébauchent et s'appuient en partie sur la prise en compte du temps long. 
La Bibliothèque Digitale Brésilienne des Thèses et Dissertations ${ }^{5}$ recense 189130 thèses de doctorat et 513667 dissertations de master soutenues au Brésil. L'accès à ce bien collectif issu, dans la grande majorité, de financements publics est libre. Les plus anciens travaux remontent aux années 1850, ceux d'anthropologie sociale à 1975 et ceux avec le mot clé ethnobotanique à 1997. Le croisement des mots clé anthropologie/ ethnologie avec végétal/plantes et la recherche sur ethnobotanique, donne un corpus de 274 (218 mémoires et 56 thèses) soutenus entre 1997 et 2020 avec un net renforcement de l'intérêt pour le thème à partir des années 2010 et une légère décroissance sur la période 2015-2019. Les années 2010-2020 représentent néanmoins près des trois quarts de ces travaux universitaires. Le contexte de dévastation environnementale dans le monde comme au Brésil, celui d'atteintes aux territoires de vie des populations traditionnelles, un relatif décloisonnement des disciplines, l'impulsion donnée par les gouvernements du Parti des Travailleurs depuis 2001 à l'ouverture de l'université à des populations marginalisées ont probablement concouru au développement de cette ligne de recherche. Cela s'illustre par l'émergence de cursus universitaires comme le MESPT (Master Professionnel en Durabilité pour les Peuples et Terres Traditionnelles) de l'Université de Brasília lancé en 2011. Ce master est conçu en alternance entre l'université et le lieu de vie de l'étudiant où sont réalisées ses activités de recherche. 73 masters professionnels (non répertoriés dans la base de l'Institut Brésilien d'Informations en Sciences et Technologie - IBICT) ont été produits dans le cadre du MESPT depuis 2011 avec deux lignes de recherche principales, les liens au territoire et les pratiques interculturelles. Cette ouverture aux populations locales sur des thèmes territorialisés faisant dialoguer diversité culturelle et diversité biologique est aujourd'hui repris par plusieurs centres universitaires.

Les 274 travaux sont issus de 50 institutions universitaires avec une forte concentration sur onze ${ }^{6} \mathrm{~d}^{\prime}$ entre elles. Trois pôles universitaires régionaux se dessinent, le nord-est (États de Paraíba et de Pernambuco), le sud (Santa Catarina et Rio Grande do Sul) et le sud-est (Rio de Janeiro et São Paulo), concentration déjà soulignée par Ritter et al. (2015). Les travaux universitaires se portent de manière équivalente sur les différents biomes sans saillance particulière ${ }^{7}$. En revanche, les approches ethnobotaniques ont été particulièrement développées, en ordre croissant, auprès des populations urbaines (7,6\%), des communautés afro-brésiliennes (10,6\%), des peuples amérindiens $(13,5 \%$, principalement les Guarani) et des communautés rurales ( $48 \%$ des travaux) mais, pour ces dernières, leur identité ou leur trajectoire historique ne sont souvent guère précisées. Ceci permet de postuler que, sur la base des quelques lignes des résumés fournis, et hormis quelques travaux d'anthropologie sociale, c'est le binôme ressourceplante / usages et savoirs, avec un fort investissement dans les plantes médicinales, qui est au centre de l'envie de recherche des étudiants et des enseignements. La diversité culturelle brésilienne, en particulier celle des populations ni amérindiennes ni quilombolas mais représentée par d'innombrables groupes culturels (vazanteiros, seringueiros, sertanejos, geraizeiros, jangadeiros ${ }^{8}$...) reconnus sur la base de leurs activités, de leur environnement ou de leur origine, semble s'estomper face à la diversité biologique. La prééminence des approches centrées sur la plante avait déjà été mise en relief par C. Diegues, sociologue de l'Université de São Paulo, qui soulignait dès 2000 combien l'ethnobiologie se liait à la conservation au sein d'une nouvelle génération de chercheurs et que, entre chercheurs naturalistes et des sciences sociales, les dialogues devaient se renforcer (Diegues et al. 2000). Une nouvelle ligne de recherche en 
ethnobotanique se renforce, en dialogue avec l'écologie historique et associe l'Université de São Paulo et différentes institutions amazoniennes (Cassino et al. 2019).

La base Lattes 9 , base des curriculum-vitae du Centre National de Développement Scientifique et Technologique ( $\mathrm{CNPq}$ ) comprend 2267 profils intégrant le terme ethnobotanique y sont recensés ( 469 en 2008, Oliveira et al. 2009) : la prédominance des doctorats issus des sciences agraires (157 sur les 1100 profils analysés) et de la botanique (313) est nette et les sciences humaines et sociales ne représentent qu'une petite fraction (53) de ces profils. La recherche sur le Web of Science ${ }^{10}$ (sur Ethnobotany and Brazil) renvoie à 855 publications avec une montée en puissance de celles-ci à partir des années 2006. Deux brèves remarques : les approches ethnopharmacologiques représentent $574(67 \%)$ des publications et un quart des articles (sur 281) est publié dans des revues brésiliennes dont Acta botanica brasilica et Rodriguesia en anglais. On ne peut clore cette brève analyse des tendances de l'ethnobotanique sans mentionner la revue Ethnoscientia lancée en 2016 à l'initiative de la Société Brésilienne d'Ethnobiologie et d'Ethnoécologie (SBEE) qui a à son actif six numéros publiés dans son actuel format. Elle a organisé le premier Symposium Brésilien d'Ethnobiologie en 1996.

\section{Conclusion}

L'exercice de décryptage de bases de données a de fortes limites liées à leur caractère malgré tout incomplet, à leur indexation face à un objet, l'ethnobotanique, aux contours non délimités dont le champ va des infinis inventaires de plantes et d'usages à des approches approfondies sur des formes de classifications des plantes. Les sources citées sont loin d'épuiser les approches ethnobotaniques au Brésil et tout un cortège d'ouvrages, souvent issus de thèses ou de mémoires, principalement en anthropologie, en ethnologie, en archéologie, en écologie historique, en génétique... analyse les rapports sociétés - monde végétal et est porteuse d'approches novatrices sans pour autant être référencés sous une étiquette ethnobotanique. Quelles lignes de recherche peut-on imaginer dans un paysage foisonnant par la diversité de ses approches et sa capacité à enthousiasmer et mobiliser une jeune génération de chercheurs? Les enjeux sont planétaires, la compréhension des rapports au monde végétal est susceptible d'étayer des droits et des reconnaissances de la diversité des formes d'être au monde. Face au désastre environnemental, l'urgence est de faire émerger de nouveaux mondes, de les faire vivre et de les faire entendre au-delà de la sphère scientifique.

\section{BIBLIOGRAPHIE}

Almeida, M. W. B. 1992 - Rubber tappers of the upper Juruá river, Brazil. The making of the forest peasant forestry. PhD Thesis. Cambridge, University of Cambridge, $376 \mathrm{p}$.

Almeida M.C. de. 2002 - António do Rosário, Frutas do Brasil numa nova, e ascetica Monarchia, consagrada à Santissima Senhora do Rosario. Apresentação Ana Hatherly (Fac-símile da edição de 
Lisboa : António Galrão, 1702), Lisboa, Biblioteca Nacional, 2002, 28 + 208 p., Nuevo Mundo Mundos Nuevos. [En ligne] URL https://doi.org/10.4000/nuevomundo.331 Consulté le 26/09/2021.

Aubertin C. 2021 - Introduction In : Aubertin C. \& Nivart A. (Ed.) La nature en partage, autour du protocole de Nagoya. Marseille/Paris, IRD Éditions/MNHN, 323 p.

Baldus H. 1968 - Bibliografia crítica da etnologia brasileira, vol. 2. Hannover, Kommissionverlag Munstermann - Druck GMBH, 868 p.

Baldus H. 1970 [1954] - Bibliografia crítica da etnologia brasileira, vol. 1. São Paulo, Kraus Reprint, 439 p.

Barretto J.-P. 2021 - Kumuã na kahtiroti-ukuse: uma "teoria" sobre o corpo e o conhecimento-prático dos especialistas indígenas do Alto Rio Negro. (Doutorado tese). Manaus, Universidade Federal do Amazonas, $190 \mathrm{p}$.

Brasil 1988 - Constituição da República Federativa do Brasil de 1988. http://www.planalto.gov.br/ ccivil_03/constituicao/constituicao.htm

Brasil 1990 - Decreto No 98.897, de 30 de Janeiro de 1990. Dispõe sobre as reservas extrativistas e dá outras providências. http://www.planalto.gov.br/ccivil_03/decreto/antigos/d98897.htm

Brasil 2000 - Lei No 9.985, de 18 de Julho de 2000. Institui o Sistema Nacional de Unidades de Conservação da Natureza e dá outras providências. http://www.planalto.gov.br/ccivil_03/leis/19985.htm

Buchillet D. 2007 - Bibliografia critica da saúde indígena no Brasil (1844-2006). Quito, Abya Yala, 614 p. Carneiro da Cunha M. 2019 - Antidomestication in the Amazon: Swidden and its foes. Hau-Journal of Ethnographic Theory 9 (1) : 126-136. doi:10.1086/703870

Carneiro da Cunha M. \& Almeida M.W.B. de. (Ed.) 2002 - Enciclopédia da Floresta, o Alto Juruá: práticas e conhecimentos das populações. São Paulo, Companhia das Letras, 735 p.

Carneiro da Cunha M., Magalhães S.B. \& Adams C. (Ed.) 2021 - Povos tradicionais e Biodiversidade no Brasil. Contribuições dos povos indígenas, quilombolas e comunidades tradicionais para a biodiversidade, políticas e ameaças. São Paulo, SBPC. http://portal.sbpcnet.org.br/publicacoes/povos-tradicionaise-biodiversidade-no-brasil/

Cassino M.F., Alves R.P., Levis C., Watling J., Junqueira A.B., Shock M.P., Ferreira M.J., Andrade V.L.C., Furquim L.P., Coelho S.D., Tamanaha E.K., Neves E.G. \& Clement C.R. 2014 - Ethnobotany and Ethnoecology Applied to Historical Ecology. In : Albuquerque U.P., Paiva de Lucena R.F., Fernandes Cruz da Cunha L.V. \& Nóbrega Alves R.R. (Ed.), Methods and Techniques in Ethnobiology and Ethnoecology. New York, NY, Humana Press, Springer : 187-208. (Springer Protocols Handbooks).

Coe-Teixeira B. 1976 - Bibliografia Comentada de Etnobotânica. Revista do Museu Paulista 23 : 41-63.

Côrrea P. \& coll. 1926-1952 [1984] - Dicionario das plantas utéis do Brasil e das exóticas cultivadas. Rio de Janeiro, Ministério da Agricultura, Instituto Brasileiro de Desenvolvimento Florestal, 6 vol.

Daniel J. Padre. 2004 [1757-1776] - Tesouro descoberto no máximo Rio Amazonas. Rio de Janeiro, Contraponto, 2 vol. : 597 et 622 p.

Dean W. 1991 - A botânica e a política imperial: a introdução e a domesticação de plantas no Brasil. Estudos Históricos 4 (8) : 216-228.

Diegues A.C., Arruda R.S., Ferreira da Silva V.C., Barboza Figols F.A. \& Andrade D. 2000 - Saberes tradicionais e biodiversidade no Brasil. São Paulo, Nupaub - USP / Probio - MMA / CNPq, 211 p. 
Emperaire L. 2021 - Gerar, cuidar e manter a diversidade biológica. In : Carneiro da Cunha M., Magalhães S.B. \& Adams C. (Ed.), Povos tradicionais e Biodiversidade no Brasil. Contribuições dos povos indígenas, quilombolas e comunidades tradicionais para a biodiversidade, políticas e ameaças, v. 7. São Paulo, SBPC, $351 \mathrm{p}$.

Fondation pour la recherche sur la biodiversité 2019 - Rapport de l'étude sur l'utilisation des données de séquençage des ressources génétiques pour l'alimentation et l'agriculture. Paris, FRB, 103 p.

Fonseca-Kruel V.S. da, Machline Silva I. \& Pinheiro C.U.B. 2005 - O ensino acadêmico da etnobotânica no Brasil. Rodriguesia 56 (87) : 97-106. https://doi.org/ $10.1590 / 2175-78602005568707$

Françozo M. 2005 - O Museu Paulista e a história da antropologia no Brasil entre 1946 e 1956. Revista de Antropologia 48 (2) : 585-612. doi.org/10.1590/S0034-77012005000200006

Hartmann T. 1984 - Bibliografia Crítica da Etnologia Brasileira, v. 3. Berlin, Dietrich Reimer, 784 p.

Kury L. 2001 - Viajantes-naturalistas no Brasil oitocentista : experiência, relato e imagem. História, Ciêncas, Saúde -Manguinhos 8 (supl.) : 863-880.

Levi-Strauss C. 1950 - The use of wild plants in tropical south America. In : Steward J.H. (Ed.) Handbook of South American Indians, vol 6. Physical Anthropology, Linguistics and Cultural Geography of South American Indians. Washington, Smithsonian Institution, Bureau of American Ethnology : 465-486.

Lisboa F.C. d. 1967 [1625] - História dos animais e árvores do Maranhão (estudo e notas do Dr. Jaime Walter). Lisboa, Arquivo Histórico de Ultramar e Centro de Estudos Históricos de Ultramar, 158 p. et fac-simile.

Marcgrave G. \& Pison W. 1648 - Historia Naturalis Brasiliae... in qua non tantum plantae et animalia, sed et indigenarum morbi, ingenia et mores describuntur et iconibus supra quingentas illustrantur. Lugdun. Batavorum, Franciscus Hackium et Amstelodami apud Lud. Elzevirium, 449 p.

Malheiros J.O. 2013 - Cristóvão de Lisboa: olhar científico e registro de imagens na produção de conhecimentos sobre a natureza do Maranhão e Grão-Pará (1624-1635). XXVIIe Sympósio Nacional de História, Conhecimento histórico e diálogo social, Natal-RN 22 a 26 de julho 2013. [En ligne] URL http://www.snh2013.anpuh.org Consulté le 24/9/2020

Morim de Lima A.G., Cabral de Oliveira J. \& Shiratori K. (Ed.) 2021 - Conhecimentos associados à biodiversidade. In : Carneiro da Cunha M., Magalhães S.B. \& Adams C. (Ed.), Povos tradicionais e Biodiversidade no Brasil. Contribuições dos povos indígenas, quilombolas e comunidades tradicionais para a biodiversidade, políticas e ameaças v. 8. São Paulo, SBPC, 280 p.

Neiva A. 1929 - Esboço histórico sobre Botânica e Zoologia no Brasil, de Gabriel de Sousa 1587 a 7 de setembro de 1922. São Paulo, Soc. Impressora Paulista, 129 p.

Neves E.G. (Ed.) 2021 - Biodiversidade e Agrobiodiversidade Como Legados de Povos Indígenas. In : Carneiro da Cunha M., Magalhães S.B. \& Adams C. (Ed.), Povos tradicionais e Biodiversidade no Brasil. Contribuições dos povos indígenas, quilombolas e comunidades tradicionais para a biodiversidade, políticas e ameaças, v. 6. São Paulo, SBPC, 87 p.

Oliveira F.C. de, Albuquerque U.P. de, Fonseca-Kruel V.S. d. \& Hanazaki N. 2009 - Advances in ethnobotany research in Brazil. Acta Botanica Brasilica 23 (2) : 590-605. doi:10.1590/ s0102-33062009000200031

Patiño V.M. 1963-1974 - Plantas cultivadas y animales domesticos en America equinoccial. T. I. Frutales, T. II. Plantas alimenticias, T. III. Fibras, medicinais, miscelaneas, T. IV. Plantas introducidas, T. V. Animales domesticos introducidos, T. VI. Plantas ornamentales. Cali, Imprenta Departamental. 
Patiño V.M. 1989 - Bibliografia etnobotanica parcial comentada de Colombia y paises vecinos. Bogotá, Instituto Colombiano de Cultura Hispanica, $371 \mathrm{p}$.

Phillips O., Gentry A.H., Reynel C., Wilkin P. \& Galvez-Durand B. 1994 - Quantitative ethnobotany and Amazonian conservation. Biology Conservation 8 (1) : 225-249.

Prance G.T., Balée W. \& Carneiro R.L. 1987 - Quantitative ethnobotany and the case for conservation in Amazonia. Conservation Biology 1 (4) : 296-310.

Ribeiro B. (Ed.). 1986 - Suma Etnológica Brasileira. v.1 - Etnobiologia. Petrópolis, Ed. Vozes/ FINEP, $304 \mathrm{p}$.

Ritter M.R., da Silva T.C., Araujo E. de L. \& Albuquerque U.P. 2015 - Bibliometric analysis of ethnobotanical research in Brazil (1988-2013). Acta Botanica Brasilica 29 (1) : 113-119. doi: 10.1590/0102-33062014abb3524

Rosário A. da O.F.M. 1702 - Frutas no Brasil numa nova e ascetica monarchia consagrada a Santissíma Senhora do Rosário. Lisboa, Officina de Antonio Pedrozo Galram, 252 p.

Sauer C.O. 1950 - Cultivated plants of South and Central America. In : Steward J.H. (Ed.) Handbook of South American Indians, v. 6. Physical Anthropology, Linguistics and Cultural Geography of South American Indians. Washington, Smithsonian Institution, Bureau of American Ethnology : 487-543.

Silva F.P.E. da. 2013 - Plantas alimentares cultivadas nas roças baniwa: mudanças e participação dos jovens. (Mestrado Dissertação). Brasília, UnB, 123 p.

Soares de Sousa G.S. 1851 [1587] - Tratado descriptivo do Brazil em 1587. s.l., Typographia universal de Laemmert, $369 \mathrm{p}$.

Staden H. 2010 [1558] - Duas viagens ao Brasil: primeiros registros sobre o Brasil. Porto Alegre, L\&PM Pocket, $181 \mathrm{p}$.

Telles Antunes M. 2011 - Saint-Hilaire e as ‘Requisições’ em Lisboa - material do Brasil e outro. Tradução e discussão de: «La Mission de Geoffroy Saint-Hilaire en Espagne et en Portugal. 1808) Histoire et Documents Par Le Dr. E.T. Hamy ». Brazilian Geographical Journal: Geosciences and Humanities research medium, Uberlândia 2 (2) : 392-464.

\section{NOTES}

1. Cette déclaration a été rédigée et promulguée au cours du $1^{\mathrm{er}}$ congrès international de la toute nouvelle Société Internationale d'Ethnobiologie. Elle est accessible en ligne: https:// www.ethnobiology.net/what-we-do/core-programs/global-coalition-2/declaration-of-belem/ 2. Les seringueiros sont les collecteurs de latex d'hévéa. L'hévéa est une espèce forestière, présente principalement en Amazonie occidentale. Chaque seringueiro parcourt deux fois par semaine, six mois par an, son estrada, soit un sentier forestier qui relie de 60 à 120 hévéas. Cette activité extractiviste se combine en général à la culture d'un ou plusieurs abattis (Almeida, 1992, Emperaire, 1997). Pratiquée dès le milieu du XIX siècle, la collecte du latex a été à l'origine de l'enrôlement des populations amérindiennes par des patrons, les seringalistas, puis du déplacement de populations originaires du nord-est du Brésil vers l'Amazonie dans des conditions aussi violentes. Actuellement, la saignée des hévéas forestiers connaît des périodes de reprise en particulier avec la production de cuir végétal mais l'essentiel de la production de caoutchouc est assuré par des plantations situées principalement dans l'État de São Paulo.

3. Quilombolas sont les communautés afrodescendantes du Brésil. 
4. https://uspdigital.usp.br/jupiterweb/obterDisciplina?sgldis=FLA0360\&nomdis= https://ppgas.museunacional.ufrj.br/disciplinas.html https://www.ppgas.ufam.edu.br/planos-de-curso.html http://www.ppginpa.eco.br/documents/ementas/ECO\%20077_Etnobiologia.pdf https://w1.solucaoatrio.net.br/somos/inpa-ppgbot/index.php/pt/disciplinas 5. Biblioteca Digital Brasileira de Teses e Dissertações (BDTD http://bdtd.ibict.br/vufind/).

6. Les 11 universités qui ont la plus importante production en termes de masters et de doctorats dans ce domaine sont l'UNESP, et l'USP (État de São Paulo), l'UnB (District Fédéral), l'UFRRJ (Rio de Janeiro), l'UFRPE et l'UFPE (Pernambuco), l'UFPB (Paraíba), l'UFSC (Santa Catarina), l'UFPEL et l'UFRGS (Rio Grande do Sul), l'INPA (Amazonas).

7. Des 274 travaux, 5,5\% concernent une problématique non spatialisée, $12,5 \%$ le Centre-Ouest soit approximativement le domaine du cerrado, 24,5\%, le Nord-Est (caatinga), $18 \%$ le Nord (forêt amazonienne), 19,5\% le Sud-Est (cerrado et forêt atlantique), $20 \%$ le sud (forêt atlantique, pampa) et pour $1 \%$ le lieu n'est pas précisé dans le résumé.

8. Vazanteiros ou varjeiros (habitants des berges des fleuves, principalement du rio São Francisco, qui pratiquent une agriculture de décrue), seringueiros (saigneurs d'hévéa en Amazonie), sertanejos (habitants des zones semi-arides dont l'économie est axée sur l'élevage du bétail), geraizeiros (habitants du nord de Minas Gerais à la limite des domaines des caatingas et de cerrados), jangadeiros (pêcheurs côtiers traditionnels du Nordeste dont les embarcations sont dénommées jangadas).

9. Le Lattes est une base de données initiée sous les auspices du CNPq en 1999. Le dépôt et l'actualisation de son CV est un passage obligé pour répondre à des appels d'offre. Elle détaille les curriculum-vitae des chercheurs, enseignants-chercheurs, étudiants de tous niveaux, de l'entrée à l'université au doctorat. Son accès est libre pour sa consultation et pour la mise en ligne de son CV. Plus de trois millions de CV y sont déposés dont environ $11 \%$ de docteurs, seule catégorie ici travaillée. Les filtres, autre que le niveau d'études, sont assez sommaires et ne permettent pas une approche fine.

10. https://www.webofscience.com/wos/alldb/basic-search

\section{RÉSUMÉS}

$\mathrm{Au}$ Brésil le nombre de chercheurs ou enseignants-chercheurs docteurs qui mentionnent l'ethnobotanique comme un des mots-clés de leur parcours est passé de 500 à plus de 2200 en douze ans. Alors que ce champ disciplinaire demeure discret en France, il explose au Brésil et mobilise des domaines très divers, disciplines naturalistes mais aussi génétique, chimie, droit, développement durable... dans un paysage territorialisé autour d'une diversité d'approches méthodologiques. Dans quelle trajectoire les savoirs sur les relations plantes-sociétés au Brésil s'inscrivent-ils ? De quels débats sont-ils porteurs ? Quels sont ses nouveaux acteurs?

In Brazil, the number of researchers and PhD-level research fellows who mention ethnobotany as one of their keywords has increased from 500 to 2,200 in the past twelve years. While this disciplinary field remains confidential in France, it is booming in Brazil and mobilizes various fields; from naturalist disciplines to genetics, chemistry, law, sustainable development... in a landscape which is the object of many different methodological approaches. Where does this 
knowledge on plant-society relations in Brazil belong? What kinds of debates does it open? Who are the discipline's new stakeholders?

INDEX

Mots-clés : ethnobotanique, Brésil, histoire, production bibliographique

Keywords : ethnobotany, Brazil, history, bibliographic production

\section{AUTEUR}

\section{LAURE EMPERAIRE}

Chercheur, IRD / UMR PALOC IRD-MNHN-SU, 01407938 27, laure.emperaire@ird.fr , Département H\&E, CP 135, 57 rue Cuvier, 75005 Paris 